\title{
Les mutations des relations entre auteurs et éditeurs littéraires à l'ère du numérique : une crise de l'autorité?
}

\author{
CORENTIN BOUTOUX \\ Université Paris Nanterre / France \\ 凶 corentin.boutoux@gmail.com \\ (iD) https://orcid.org/0000-0001-8295-9685
}

RÉSUMÉ. Depuis l'apparition de la figure moderne de l'éditeur au début du XIXe siècle, la nature des relations entre les auteurs et le champ de production des œuvres littéraires a été le fruit d'innombrables débats. Entre le créateur et celui qui transforme l'œuvre en produit commercialisable, qui détient quelle autorité? La transformation de l'édition en industrie culturelle semble avoir relégué l'auteur à la seconde place et l'avoir inscrit dans une longue histoire de domination, tout en étant l'instigateur de sa légitimité et le moteur de sa reconnaissance. Le développement de l'informatique et des technologies numériques entraîne de nouvelles mutations du marché du livre. Alors que les acteurs institués adoptent progressivement le recours à ces nouveaux outils pour entériner leur domination, des circuits parallèles se mettent en place, à la périphérie du pouvoir. À I'heure actuelle, que devient le rapport de cet auteur à ces constructions instituées? Sa place est-elle renégociée? 
RESUMEN. Las mutaciones de las relaciones entre autores y editores literarios en la era digital: ¿una crisis de la autoridad? Desde la aparición de la figura moderna del editor a inicios del siglo XIX, las relaciones entre autores y el ámbito de producción de las obras literarias ha sido objeto de numerosas controversias. ¿Entre el creador y el que transforma la obra en un producto comercializable, quién dispone de autoridad? A pesar de que el autor ha sido el instigador de su legitimidad y el motor de su reconocimiento, transformar la edición en una industria cultural puede relegar al autor a un segundo plano y la vez a un largo periodo de dominación. El desarrollo de la informática y de las tecnologías digitales provoca paulatinamente nuevos cambios en el mercado del libro, los actores establecidos utilizan poco a poco estas nuevas herramientas para confirmar su poder mientras que otras dominaciones se establecen en la periferia del poder. Por tanto ¿qué ocurre actualmente entre el autor y las construcciones instituidas? ¿Se vuelve a negociar la plaza que debe ocupar el autor?

ABSTRACT. The mutation of relationships between authors and publishers of literature in the digital era: a crisis of authority? Since the first appearance of the publisher's modern character at the beginning of the $19^{\text {th }}$ century, the relationship between authors and the field of production of literary works has been the subject of numerous debates. Between the creator, and the one who transforms the work in a marketable product, who detains which authority? The transformation of publishing into a cultural industry seems to have relegated the author to a secondary place, putting him in a long story of subjugation, while this first still being the instigator of his legitimacy and recognition. The development of computer science and digital technologies generates new mutations of the book market; whereas the established actors gradually employ these new tools to confirm their domination, parallel paths appear at the power's periphery. Nowadays, what has become of the relation between the author and these institutions? Has his position been negotiated again?
PALABRAS

CLAVE:

autoridad;

autor;

edición;

literario;

digitalización

KEYWORDS:

authority;

author;

publishing;

literature;

digital 


\section{Introduction. Histoire d'une longue domination}

Les années 1950 annoncent les premières étapes de lourdes transformations dans le milieu du livre en France, qui se poursuivront tout au long des décennies suivantes. Parmi les évènements les plus marquants de cette période surviennent le rachat de Denoël par Gallimard en 1951, et celui de Grasset puis de Fayard par Hachette en 1954 et 1958 respectivement. Ces quelques phénomènes de récupérations ponctuelles sont motivés par des impératifs autant symboliques - celui de ne pas voir disparaître des maisons rendues exsangues par la guerre - quéconomiques - celui de récupérer le catalogue de ces structures :

Il s'agit pour certains, qui prennent acte de la déstabilisation de nombreuses entreprises dédition, consécutives au conflit mondial et aux difficultés économiques de l'aprèsguerre, de stabiliser des réseaux d'auteurs et d'appuyer le développement de leur maison par une politique de fonds. (Piault, 1992, p. 630)

À leur suite, les années 1960 signent les débuts d'une phase de concentration dans le secteur de l'édition, à travers toute une série de rachats et de fusions se poursuivant toujours, sinon plus, à l'heure actuelle. Cette restructuration profonde du paysage éditorial donne rapidement lieu à un partage du marché tenu essentiellement par une minorité de grands acteurs, à qui sont affiliés - ou bien autour desquels gravitent - un nombre bien plus élevé d'entreprises de tailles plus modestes (Piault, 2018)'1. Ce modèle sera qualifié dès le début des années 1980, au sujet du monde du livre, d'« oligopole avec franges » (Reynaud-Cressent, 1982, p. 61). Le changement de paradigme qui s'instaure laisse dès lors percevoir, de manière sans cesse plus flagrante, lorientation des grands groupes contrôlant ce marché vers une rationalisation de leur production : la préférence est donnée à la recherche du profit et moins à la découverte, plus volontiers laissée aux acteurs moins puissants mais qui peuvent en contrepartie orienter leur politique « vers la recherche de nouveaux talents ou de créneaux encore inexploités » (Benhamou, 2003, p. 99). Toutefois, les transformations peuvent aussi bien s'opérer à l'échelle microéconomique : l'accroissement de la taille d'une entreprise implique fréquemment la complexification de sa hiérarchie et la portée des moyens quelle peut mettre en place, entraînant l'installation de régimes de visibilité variables, le succès appelant le succès et le profit appelant au développement (Bouvaist, 1991, p. 47).

${ }^{1}$ Dans le secteur de la littérature, la position de tête est occupée par Hachette, sans que cette première place n'ait jamais été sérieusement inquiétée. Loin derrière mais pesant lourdement sur le marché se trouvent le groupe Editis, le groupe Madrigall (Gallimard/Flammarion), Média-Participation, le groupe Albin Michel. Parmi les puissants, seul Lefebre-Sarrut ne publie pas de littérature. 
Mais quoique le milieu de l'édition ne soit pas homogène - loin s'en faut -, de nombreuses pratiques restent partagées entre les structures comme en témoigne, entre autres, l'existence d'une convention collective (Syndicat National de l'Edition, 2000). Ces similitudes se constatent par-delà les spécificités propres à chaque entreprise, et indépendamment de son importance sur le marché ou de sa place dans l'histoire culturelle, indépendamment aussi de ses politiques auctoriale et éditoriale. Le modèle de lédition « traditionnelle » qui traverse ces différences tend globalement à suivre ce qu'on appelle la "chaîne du livre " : cette figure métaphorique symbolise les rapports entretenus entre chacun des acteurs qui composent à la fois l'entreprise et ceux externes à elle, mais avec qui cette entreprise entretient néanmoins des relations complexes. Le concept de «chaîne » induit éminemment la notion d'interdépendance des différents maillons qui la constituent : l'absence d'un seul d'entre eux rend potentiellement la chaîne non fonctionnelle. Elle induit également la notion de déroulement : une chaîne possède un début et une fin (pour peu quelle ne soit pas circulaire) mais aussi un principe d'immuabilité de la position de ces maillons. Lenjeu est donc de comprendre en quoi les relations entre les deux éléments fondamentaux que sont l'auteur et l'éditeur, au sein de cette chaine du livre, tendent potentiellement à être remises en question à l'heure de l'émergence et de l'utilisation des nouvelles technologies. En effet, ce modèle institué entraîne un rapport de domination le plus souvent au désavantage de l'auteur, et engendre en conséquence une crise de la confiance à plusieurs égards : confiance en le bien-fondé du fonctionnement de ce système tout entier, mais aussi confiance en la légitimité même de certains des acteurs à agir. Les nouvelles technologies de l'information et de la communication amènent chacun à reconsidérer sa position respective, et de fait la nature de ses liens avec les autres composants de la chaîne, que ceux-ci se trouvent originellement en amont ou en aval de soi. Certains, voulant stabiliser leur prédominance, cherchent à exploiter ces outils comme une démultiplication des moyens d'emprise dont ils disposent déjà. D'autres, dans une recherche d'affranchissement, tentent d'agir aux marges du système, et cherchent dans ces outils un moyen alternatif d'acquérir une légitimité, voire une autorité. Toute la question est donc de voir si, dans cette ère du numérique, cette autorité est bien en «crise » ou non. Il est à noter que les réalités propres à plusieurs secteurs de l'édition (comme l'édition scolaire, juridique ou scientifique) répondent à des problématiques et des enjeux qui leurs sont spécifiques ; à cette fin, les considérations avancées ici ne porteront que sur la littérature (dans sa définition ou dans les formes que prend sa consécration) et son traitement par le milieu éditorial, lorsque celui-ci dédie son activité à ce champ. 
L'analyse présentée ici est notamment exemplifiée par des citations provenant d'une série d'entretiens semi-directifs réalisés au cours des années 2019-2021. Ces échanges ont été menés auprès de vingt-cinq acteurs du monde du livre, imprimé et numérique, de sorte à fournir un aperçu des diverses pratiques auctoriales et éditoriales contemporaines. Les questions, au nombre d'une trentaine en moyenne, ont été adaptées en fonction de la nature des interlocuteurs. Des extraits de leurs déclarations sont reproduites dans cet article sous la formulation « entretien avec l'auteur ».

\section{Qu'est-ce que l'autorité « littéraire » ?}

\subsection{L'autorité comme délégation}

Afin de comprendre ce que recouvre le concept de « crise de l'autorité » dans le milieu littéraire que nous étudions ici, il convient de commencer par rappeler ce qu'est l'autorité. Dans La Crise de la culture, Hannah Arendt définit celle-ci par négation de ce qu'elle n'est pas, soit « le pouvoir» et « la violence » (Arendt, 1972A, p. 123). En séparant l'autorité de l'usage de «moyens extérieurs de coercition et de contrainte » (idem), le pouvoir se comprend pour sa part comme l'application effective de ces moyens, ou la capacité à les mettre en action. À l'inverse, l'autorité nécessite que celles et ceux « dont l'obéissance est requise la reconnaissent inconditionnellement ; il n'est en ce cas nul besoin de contrainte ou de persuasion » (Arendt, 1972B, p. 146). À la suite de cette constatation, deux déductions se présentent. La première repose sur le lien ténu qui se dessine entre pouvoir et autorité : comme le rappelle Hannah Arendt dans un autre de ses textes, "[...] power springs up between men when they act together and vanishes the moment they disperse ${ }^{2}$.» (Arendt, 1998, p. 200). Ainsi, le pouvoir est avant tout un concept relationnel et intersubjectif, et ceci indépendamment de l'usage concret qui en est fait ou non ; par conséquent, la présence d'autrui est « sa condition même » (Sintomer, 1994). Cette conception n'est toutefois pas nouvelle : elle se retrouve dès L'Encyclopédie, où le pouvoir est définit comme «Le consentement des hommes réunis en société.» (Jaucourt, 1765, p. 255). L'autorité, nécessairement hiérarchique du fait de cette « obéissance inconditionnelle » qui l'instaure, devient conséquemment elle aussi relationnelle et intersubjective. La deuxième déduction repose sur la suite logique de cette première consi-

2 «Le pouvoir jaillit parmi les hommes lorsqu'ils agissent ensemble et retombe dès qu'ils se dispersent». "Power» est quelquefois traduit par "puissance» dans cette citation (Quelquejeu, 2001) ce qui nous semble ici un faux-sens. Nous voyons plus de justesse dans la traduction qu'adopte notamment Sintomer (Sintomer, 1994). 
dération : l'autorité induit manifestement l'existence d'un rapport inégalitaire entre les parties qui en disposent, et celles n'en disposant pas. Cependant, ce rapport déséquilibré repose sur l'acceptation et le consentement des sujets en position dominée ; dans le cas contraire, cette autorité serait violence. Par-delà la dynamique de soumission, l'autorité repose donc sur le principe de reconnaissance et de légitimité de son rôle et de son action. L'autorité se comprendrait dès lors comme la forme déléguée du pouvoir, ou sa représentation, autrement dit son étendard fonctionnant à la fois comme une capacité de dissuasion et de conviction de son bien-fondé. L'autorité, à l'instar du pouvoir qui la permet, ne fonctionne que tant qu'elle est elle-même perçue comme légitime. C'est ce que Max Weber formule déjà dans Economie et société, lorsqu'il donne une définition de la «domination » dans son caractère rationnel, comme « reposant sur la croyance en la légalité des règlements arrêtés et du droit de donner des directives qu'ont ceux qui sont appelés à exercer la domination par ces moyens. »(Weber, 1995, p. 289).

C'est selon cette acception du pouvoir, et de l'autorité qui en est la forme déléguée, que s'aborde le concept particulier « d'autorité » dans le champ littéraire. Celle-ci renvoie à un constat double. D'abord, fondamentalement, elle renvoie à la revendication exprimée par certains acteurs de ce champ d'une capacité à dire, à un moment donné de la vie d'une œuvre ou d'un ensemble d'œuvres, en quoi cette œuvre relève ou non du « littéraire », et est donc porteuse de la caractéristique de "littérarité » (Jakobson, 1973, p. 15). Dans ce contexte, l'autorité, c'est déclarer « ceci est une œuvre littéraire » ou au contraire refuser de le déclarer; c'est aussi supposer la légitimité de sa posture qui amène à agir de la sorte, et c'est chercher à faire en sorte que cette attestation soit supportée et reconnue par la suite par le tissu relationnel qui compose la « chaîne du livre ». Ensuite, cette autorité renvoie au statut même des individus et à leur fonction au sein d'un espace social spécifique, celui du « milieu littéraire ». Ce milieu se définit comme la somme des sources de légitimation et de reconnaissance; il se constitue notamment des "gens de lettres » et les rapports qui s'y observent sont régis par des réseaux à la fois économiques et symboliques. On y retrouve les éditeurs bien sûr, mais aussi les critiques, les jurys de prix littéraires, les libraires même ; autrement dit, toute la masse des acteurs du monde du livre dans sa complexité où chacun, en supposant la légitimité de sa propre posture, cherche aussi à exprimer une capacité à valoriser l'œuvre et son auteur. Comme le rappelle Bourdieu dans « Le champ littéraire » : «Un des enjeux centraux des luttes littéraires est [...] le monopole du pouvoir de consécration des producteurs ou des produits » (Bourdieu, 1991, p. 14). Faire autorité, incarner une autorité : c'est dans cet espace double que vont se jouer les rapports de force dont semble, à priori, être exclu le grand public en bout de chaîne. Bien que ce dernier dis- 
pose du pouvoir de ne pas acheter, de ne pas lire, il n'en est pas moins réduit au rôle du consommateur sur lequel s'exercent tout un ensemble d'autorités sans qu'il ne paraisse en disposer vraiment lui-même.

\subsection{L'ascendance de l'autorité éditoriale}

Dans ce champ, c'est à une figure des coulisses, « homme de l'ombre » selon la formule de François Dosse (Dosse, 2014), méconnu de ce grand public, que nous nous intéressons particulièrement. L'amalgame est fréquent entre l'« éditeur » conçu comme un individu singulier disposant de certaines responsabilités au sein d'une structure éditoriale, et l'« éditeur » conçu comme cette structure dans son entièreté. Cette confusion est encore plus manifeste dans le cas des entreprises de petite taille, lorsque la presque totalité des responsabilités (éditoriales comme décisionnelles à l'égard de l'entreprise entière) peut échoir à une seule personne. Afin de bien distinguer les deux, nous préférerons désigner le premier comme « individu-éditeur » (qui désigne alors l'autorité déléguée par cette instance structurelle, même dans le cas d'une société unipersonnelle), et le second comme « instance éditoriale » (qui désigne alors l'ensemble des éléments qui constituent le pouvoir, par-delà les variations structurelles. Le rôle de l'individu-éditeur se bâtit en premier lieu en amont de toute mise à disposition du milieu littéraire comme du public. Il s'accomplit en effet par la supposition de sa légitimité à publier et à reconnaître la littérarité (ainsi que l'« opportunité » qu'il estime suffisante pour entreprendre la publication), puis par la mise en œuvre des moyens permettant cette publication. L'individu-éditeur précède les autres maillons de la chaîne de la reconnaissance; en cela, il est le premier véhicule du capital symbolique qui sera conféré à l'œuvre, ce qui permettra d'en faire une œuvre dite « littéraire ». Il est également le premier véhicule du capital symbolique qui sera conféré à l'auteur, ce qui lui permettra de se revendiquer comme "auteur de littérature », autrement dit comme écrivain, "premier rang de dignité parmi les hommes de Lettres»(Viala, 1985, p. 280). En qualité de premier récepteur professionnel, et de premier lecteur du texte au sein de la chaîne du livre ${ }^{3}$, cet individu-éditeur se voit guidé par un « horizon d'attente », concept dont Jauss donne les « trois facteurs principaux » :

3 La première sélection peut être un rôle également endossé par le service des manuscrits (comme c'est le cas d'Actes Sud), ou d'un comité de lecture (le plus célèbre étant celui de Gallimard). Dans ces cas-ci, la première lecture est en effet assurée par ces éléments, qui exercent alors leur «horizon d'attente» relativement à l'œuvre. Ces services et comités peuvent agir dans le sens d'un refus avant même que le manuscrit ne soit présenté à un individu disposant de responsabilités éditoriales et ainsi empêcher, en amont, la découverte et la publication d'un auteur (Deguy, 1988, p. 19). Toutefois, en dépit de cette influence, la décision finale de publication ne leur revient pas. 
[...] L'expérience préalable que le public a du genre dont elle [l’œuvre] relève, la forme et la thématique d'œuvres antérieures dont elle présuppose la connaissance, et l'opposition entre langage poétique et langage pratique, monde imaginaire et réalité quotidienne. (Jauss, 1978, p. 54)

La lecture de Jauss éclaire sur les motivations qui amènent un individu-éditeur vers la décision de publication : celle-ci se fait, d'abord, sur l'estimation de la correspondance de l'œuvre considérée vis-à-vis de la ligne éditoriale que cet individu établit (ou bien qui est établie avant lui et dont il s'accommode pour y répondre ou la faire évoluer). Elle se fait ensuite relativement à ses préférences esthétiques, sans que la justification littéraire ne soit toujours précisément définie. Aux éditions Premier Parallèles, cette attente correspond au « sentiment que le projet à une force, un poids ; qu'il dit quelque chose, et qu'il le dit d'une manière qui est singulière, unique. » (entretien avec l'auteur). Hubert Nyssen, directeur d'Actes Sud de 1978 à sa mort en 2011, évoque pour sa part le « plaisir et la nécessité ${ }^{4}$ »(Editions Actes Sud, s. d.). Bertrand Fillaudeau, directeur des éditions Corti, mentionne quant à lui :

S'il s'agit d'un auteur que nous avons déjà commencé à publier, [nous attendons de lui] qu'il trace son sillon tout en continuant à nous surprendre. Si c'est un « arrivant ", c'est à la fois parce que nous avons découvert un texte qui nous a séduit et qui ne ressemblait à aucun autre, et que nous sentons que cet écrivain est plein de promesses. (Entretien avec l'auteur)

Par sa capacité à reconnaître la littérarité préalablement supposée par l'auteur, l'individu-éditeur de littérature confère au livre un double privilège : l'entrée non seulement dans l'histoire littéraire de la maison d'édition, mais aussi celle dans l'histoire littéraire plus globale d'un espace linguistique partagé. L'autorité éditoriale est ce qui fait de l'individu-éditeur le représentant de son catalogue, de son histoire, de sa conception de la littérature. Ce travail, malgré tout, devra ensuite être confronté à une attestation de la validité de toute cette entreprise par des instances tierces, considérant l'œuvre et son auteur et, par extension, le travail éditorial qui a été accompli sur cette œuvre. La légitimité présupposée doit être effectivement reconnue.

Remarquons cependant que par-delà ses caractéristiques personnelles, l'individu-éditeur reste une entité fluctuante selon le milieu où il évolue. Il est parfois décisionnaire dans la société, parfois simple exécutant, parfois libre et parfois soumis luimême à une hiérarchie interne. Mais dans tous les cas, il reste l'intermédiaire principal

4 La formule originelle remplaçait « nécessité » par " gravité » (Sadowska-Guillon, 1988, p. 125), terme renvoyant moins à une image idéalisée de la littérature, et qui ne fut plus utilisé par la suite. 
entre l'auteur et la structure au nom de laquelle il parle et dont il se fait le porte-parole, incarne le contact privilégié de chacun des auteurs dont il a la responsabilité des publications $^{5}$. C'est dans le cadre des relations incluant l'auteur que la distinction des statuts trouve toute son importance : l'auteur, individu singulier (du moins contractuellement parlant, même si plusieurs individus sont à l'origine d'un manuscrit unique), est en contact avec son éditeur comme individu lui aussi singulier, et ce de manière indépendante des relations qu'il peut, plus ponctuellement, entretenir avec le service de presse ou le service juridique ${ }^{6}$. Mais par-delà les spécificités propres à chaque structure - et qui peuvent apporter quelques nuances à ce qui a été exposé jusqu'à présent -, le double dénominateur commun à son action demeure essentiellement celui de «faire le livre », puis de « faire vivre le livre ».

\subsection{L'autorité auctoriale face au verrouillage juridique}

À ce cadre symbolique, qui entérine l'ascendance de l'instance éditoriale comme de l'individu-éditeur sur l'auteur, s'ajoute le fonctionnement de tout un appareil de loi. Si celui-ci a été originellement institué pour défendre les droits de l'auteur, il confirme aussi le déséquilibre de la relation étudiée. Au regard du droit français, aucune définition précise du métier d'éditeur n'existe ; comme l'indique le Code de la propriété intellectuelle, ce statut n'est acquis que par le truchement de l'établissement d'un contrat d'édition d'un auteur à « une personne appelée éditeur » (article L132-1), autrement dit par l'engagement du processus de publication reconnu par les deux signataires. À l'inverse, le statut d'auteur est clairement décrit : l'article L133-1 dispose ainsi que « la qualité d'auteur appartient, sauf cas contraire, à ceux sous le nom de qui l'œuvre est divulguée ». Cette paternité dans l'acte de création donne lieu à tout un ensemble de droits, dont certains sont cessibles - comme les droits d'exploitation -, et d'autres non, comme les droits moraux qui engagent notamment le droit au respect de son nom, de sa qualité d'auteur, et de son œuvre (article L121-1). Le fondement de l'autorité de l'auteur, relativement à sa création, provient de l'originalité de l'œuvre

5 Cette responsabilité peut être endossée par un agent littéraire lorsque l'auteur y a recours; ce modèle éditorial, auquel les anglo-saxons sont habitués, reste peu fréquent en France, et l'apanage de quelquesuns, souvent perçu comme un privilège réservé aux auteurs «best-sellers ».

6 Selon le schéma le plus usuel, la variété des responsabilités concentrées par un seul individu est inversement proportionnelle à la complexité hiérarchique de l'entreprise. Ainsi, lorsqu'il est question de petites structures, il est fréquent de voir l'individu-éditeur endosser des responsabilités périphériques à ses fonctions premières et assumer les tâches dévolues ailleurs au représentant, au maquettiste, voire, plus rarement, au fabricant. 
(qui confirme sa paternité), et de la responsabilité de son créateur vis-à-vis de celle-ci. Toutefois, cette autorité est entièrement indépendante de toute hiérarchie de valeur, et de l'interrogation relative à la littérarité de l'œuvre. L'« autorité » de l'auteur est donc à double facette : en qualité de personne physique signataire du contrat, son autorité est la conséquence d'une " autorisation ", celle du système légal qui permet à l'auteur de se dire tel. En qualité de créateur d'une œuvre de l'esprit, son autorité est le produit d'une construction culturelle et sociale complexe, qui n'existe pas sans l'œuvre. Que la littérarité soit supposée par l'auteur, ou bien qu'elle soit reconnue par d'autres, cela ne relève pas de compétences juridiques. Monique Nemer résume ainsi :

La décision éditoriale statue sur l'auteur et non sur l'écrivain [...] pour un éditeur, l'auteur est une notion qui ne souffre pas de degré d'intensité - on n'est pas «plus ou moins » auteur, comme une statue n’est pas « plus ou moins » équestre... (Nemer, 1996, p. 186)

La complexité des relations entre l'auteur et l'éditeur provient alors essentiellement du degré de reconnaissance, par l'individu-éditeur, de la littérarité que l'auteur suppose potentiellement sur son œuvre. Elle provient aussi de la reconnaissance, par l'auteur, de l'existence de critères économiques et esthétiques justifiant potentiellement le refus de publication. En cas d'acceptation de publier, l'entérinement de la relation entre un auteur et son éditeur est permis par l'existence de l'entité qui les relie : l'œuvre. Les rapports formels d'exploitation, autrement dit la nature des droits cédés et les conditions de cette exploitation, prennent essentiellement la forme de ce qu'on nomme un contrat d'édition. On comprend par «contrat d'édition » un contrat dit à " compte d'éditeur », soit lorsque l'instance éditoriale s'engage à faire rentrer l'œuvre dans la matérialité et à garantir sa distribution à ses frais. Le droit français refuse cette appellation à toutes les autres formes contractuelles (notamment les contrats dits « à demi » et les contrats dits « à compte d'auteur »). Lorsque ce contrat est établi, «l'obéissance inconditionnelle » arendtienne de l'auteur à l'autorité éditoriale devient factuelle; en dehors du cadre du contrat (avant signature de ce dernier, ou suite à sa rupture), le rapport relationnel est formellement inexistant, et n'implique pas directement, à priori, le concept de hiérarchie et donc d'autorité.

À de multiples égards, la transformation du monde du livre contemporain depuis soixante-dix-ans se comprend comme un verrouillage : la posture dominée dans laquelle se trouve l'auteur résulte de facteurs aussi bien structurels que légaux. Ce dernier est " atomisé ", isolé face au système institutionnel dual, étatique et éditorial ; le livre est un produit commercialisable inscrit au sein d'un ensemble de logiques marchandes régulées sur lequel il n'a qu'un contrôle très limité. L'auteur et l'éditeur, 
toutefois, restent dans un rapport de mutualité nécessaire : l'éditeur ne peut vivre sans les créations de l'auteur ; l'auteur, pour sa part, est engoncé dans un engrenage le poussant à considérer que sa soumission à l'instance éditoriale est une étape nécessaire pour pouvoir accéder à la reconnaissance, ou tout du moins à la visibilité. Cette allégeance, à priori indéboulonnable, trouve ses racines dans l'établissement progressif d'un régime de la croyance en le bien-fondé de cet assujettissement. En dépit de ce verrouillage, l'environnement lui-même est en constante mutation; les évolutions technologiques les plus contemporaines, qui induisent la dématérialisation de la production comme des rapports entre les maillons de la chaîne, sont-ils mis à mal ? Se voient-ils au contraire renforcés? Il s'agit donc maintenant de voir si l'arrivée des technologies numériques remet en cause ce déséquilibre manifeste qui a été constaté, si le pouvoir et l'autorité se définissent toujours selon les mêmes procédés, ou prennent d'autres formes.

\section{Les espaces d'expression numérique et la crise de l'autorité de l'auteur et de l'éditeur}

3.1. L'autorité éditoriale dans l'espace numérique

Le développement des nouvelles technologies permet de sortir des contraintes propres au cadre physique : tout en demeurant un objet matériel, le livre dédouble son existence. Celui-ci dispose désormais d'une identité dématérialisée, essentialisée en un nombre restreint de métadonnées permettant son identification, son classement, et facilitant de fait sa marchandisation. Ces métadonnées se conçoivent comme des informations péritextuelles réinvesties dans le contexte informatique. D’un point de vue économique et logistique, ces marqueurs d'identification se présentent comme une démultiplication des informations exploitées à des fins comptables, puis partagées entre professionnels (notamment entre structures éditoriales et imprimeurs ou distributeurs, puis entre distributeurs et libraires pour assurer la continuité de la production et du réassort des titres en librairie). L'informatique, qui offre la possibilité d'immédiateté de partage de ces informations entre tous les maillons de la chaîne du livre, permet de fait une "nouvelle logique d'entreprise " (Bouvaist, 1991, p. 15) et d'optimiser la rationalisation de la production. Mais au-delà de ces considérations économiques, les nouveaux outils sont aussi un moyen d'optimiser la mise à disposition d'informations ciblées à destination du public profane.

Ainsi, bien que tous n'en disposent pas, la plupart des maisons d'édition possède désormais un site Internet dédié ; la fondation des premiers est tardive, et remonte à la fin des années 1990. Mais de ces premières tentatives jusqu'à aujourd'hui, 
les fonctions de ces sites sont restées identiques ; la plus essentielle d'entre elles tient en la présentation du catalogue, portant à la fois sur le fonds et les nouveautés : le but premier est, ainsi, de remplacer le catalogue papier à destination du grand public. Ces sites n'endossent pas la fonction, la plupart du temps tout du moins, de plateformes de vente mais sont de simples relais promotionnels. La commercialisation des œuvres à proprement parler y est assurée par des espaces spécialisés, librairies en ligne ou sites d'e-commerce généralistes, et vers lesquels des liens renvoient ${ }^{7}$. Conséquemment, le rôle assuré est essentiellement celui de la vitrine : les informations fournies par l'exploitation de métadonnées portent à la fois sur la disponibilité des titres et les moyens de leur identification. Au-delà de cette fonction, les sites assurent également le relais de la parole promotionnelle tierce, notamment par la mise à disposition de dossiers de presse ou d'interviews. Le catalogue en ligne, s'il représente d'ordinaire la plus large portion d'un site, est aussi fréquemment accompagné d'informations à destination des auteurs (relatives aux conditions d'envoi des manuscrits), et des lecteurs (relatives au calendrier des évènements à venir).

L'espace numérique ne se limite cependant pas à la simple transmission d'informations commerciales ou de nature promotionnelle; il est aussi le moyen de produire un nouveau tissu symbolique. Ainsi, le site-vitrine dessert le second objectif de médiatiser une parole éditoriale dématérialisée. Par la présentation de son historique jalonné d'éléments-clés, souvent relatifs à la publication d'œuvres ou d'auteurs emblématiques ; par l'évocation de la consécration de ces derniers suite à l'attribution de prix littéraires prestigieux; par la mention de dates notables de l'histoire de la maison, le site incarne un vecteur de légitimité éditoriale dont le but est la mythification de la structure tout entière. Dans son ouvrage cité supra, Jauss définit au sujet de la poésie la «mythologie » comme ce qui « se mesure [...] à l'idée, ou à l'image que le lecteur se fait de la réalité représentée, et non pas à la connaissance objective qu'il en a. » (Jauss, 1978, p. 289). Dans la poursuite de cette réflexion que nous ne restreignons pas à l'art poétique, le principe de «mythification » laisse deviner un phénomène constant, ou plus précisément en perpétuelle production, qui permet d'entretenir un imaginaire. Celui-ci résulte donc aussi bien d'une création en aval que d'une réception en amont. Ainsi, dans le contexte de l'édition, la mythification correspond, d'abord, à l'image que l'entreprise produit à son propre sujet et qu'elle véhicule. Elle correspond, ensuite, à l'image effective que le lectorat se forge vis-à-vis de cette entreprise, image possiblement influencée par

7 Exception notable à ce phénomène, le groupe Harper Collins dispose d'un système de vente directe, permettant selon certaines analyses de «mieux contrôler ses canaux de vente» (Oury, 2014). 
l'action mythifiante. Par le biais du storytelling récapitulant l'évocation des grands succès d'une maison et le rôle que des figures emblématiques purent jouer dans ces succès, le site présente un discours éditorial à la fois fixe et unifié : le personnage-éditeur comme représentant de l'autorité est désincarné, et disparaît dans une parole singulière qui estompe les individualités. Le site de Fayard écrit par exemple, dans un texte de présentation non signé :

Une grande maison a besoin de constance et de continuité dans sa ligne éditoriale ; dans notre verger, certains fruits sont longs à mûrir, et une longue préparation du terreau est nécessaire à l'acclimatation des plus beaux plants, puis aux plus prometteuses récoltes. (Éditions Fayard, s. d.)

La parole éditoriale unifiée tend à effacer les spécificités de l'individu-éditeur singulier, dissimulant celles-ci soit derrière la définition des valeurs de la maison, soit derrière la figure, également mythifiée, de l'éditeur-fondateur. Ces phénomènes dépassent les particularités propres à la nature de chaque structure et s'observent aussi bien place à l'échelle du petit éditeur indépendant (comme Minuit), de l'éditeur-maison mère d'un plus grand groupe (comme Actes Sud), qu'à l'échelle des sites appartenant à des groupes encore plus larges (comme Editis). Ce lissage dans l'énonciation est aussi celui qui se retrouve à l'échelle du catalogue : l'harmonisation des métadonnées dans l'espace numérique tout entier, qui facilite l'identification des œuvres, tend en contrepartie à atténuer, voire effacer des caractéristiques propres aux œuvres ou aux auteurs, qui pourraient autrement constituer des marques de leur autorité auctoriale. C'est ainsi que si, selon Gérard Genette, $\grave{A}$ la recherche du temps perdu, de Marcel Proust, "ne comporte aucune indication générique, et cette discrétion s'accorde parfaitement au statut fort ambigu d'une œuvre à mi-chemin de l'autobiographique et du romanesque »(Genette, 1987, p. 100), l'ouvrage se trouve malgré tout catégorisé en " romans et récits » sur le site de Gallimard (Editions Gallimard, s. d.). Cette constatation s'applique d'ailleurs aussi bien à l'édition de poche qu'aux éditions complètes, dans les collections Quarto et de la Pléiade. Dans un esprit similaire d'uniformisation, Frères sorcières d'Antoine Volodine, auquel s'accole le véritable hapax d'estampillage « Entrevoûtes » sur la première de couverture et la page de titre, se retrouve sous la bannière bien plus indistincte de « roman » sur le site du Seuil (Éditions du Seuil, s. d.).

Le site comme vitrine, ainsi, se conçoit comme un espace de promotion à moindre coût (en ce qu'il n'induit pas d'impératifs financiers liés à l'occupation de l'espace physique ou médiatique), et éloigné d'un objectif d'archivage qu'il pourrait pourtant remplir. Cette portée promotionnelle amène le site à s'orienter plus volontiers sur la nouveauté que sur le fonds, considération nétant pas sans entraîner cer- 
tains écueils. Chaque site révèle ainsi une part d'incomplétude, parfois considérable, que les informations manquantes soient relatives aux auteurs ou aux ouvrages. En outre, la politique même de numérisation des œuvres par les différentes structures éditoriales s'inscrit dans une réflexion similaire, visant avant tout une proposition simultanée des derniers ouvrages parus qu'à une campagne de remise à jour de titres plus anciens, à fortiori si ceux-ci ne sont pas au panthéon des "grands noms » de la maison. Cependant, l'esthétique de renouvellement permanent n'impacte pas le déséquilibre entre les têtes d'affiche et les auteurs «secondaires ", disposant d'une moindre couverture médiatique. Le site Internet est un espace de concentration de la parole tierce promotionnelle réalisée ailleurs, mais pas un espace d'investissement. Comme le rappellent Peltier et Moreau, «Très rares sont les œuvres à bénéficier d'une forte promotion en ligne sans avoir déjà une forte présence dans les médias traditionnels »(Peltier et Moreau, 2016). Les auteurs invisibles le restent, entérinant même avec l'apparition de ces nouveaux moyens de promotion le principe selon lequel le succès engendre le succès.

Sur ce dernier point, il reste néanmoins important de préciser que les stratégies varient grandement d'une entreprise à l'autre : tandis que certaines maisons sont des pure players, comme les Éditions du 38 ; d'autres sont très peu portées sur la question numérique, comme les Éditions Corti qui ne comptent que sept ouvrages dans ces formats, sur les presque huit cents inscrits au catalogue. Toutefois, quel que soit l'engagement particulier envers le numérique de chacune des structures éditoriales publiant de la littérature, les modèles de production révèlent une nette prédominance de l'homothétie dans la forme des ouvrages numérisés, soit d'une conceptualisation de ces ouvrages comme copies aussi conformes que possible de leurs pendants imprimés. Il faut coupler à ces constatations le constat de l'échec relatif du livre numérique en France, tout du moins en ce qui concerne la littérature : en 2018, celle-ci représentait $4,81 \%$ de la part des ventes numériques dans les ventes totales des éditeurs (contre 35,98\% pour le secteur professionnel et universitaire). L'édition numérique dans son ensemble, la même année, représentait 5,1\% du chiffre d'affaires de l'édition (Politis Boublis, 2019, p. 9-10). Plusieurs pistes d'explication se dessinent, parmi lesquelles le manque d'intérêt du grand public, ancré dans ses habitudes, ou encore le caractère prohibitif du prix des ouvrages dématérialisés, considérés par beaucoup comme trop proche de celui de l'imprimé. Arnaud Nourry, PDG de Hachette Livre, déclarait ainsi en 2016 : « Le consommateur ne voit visiblement pas l'intérêt de payer plus cher en achetant une machine pour lire un livre pas tellement différent de celui qu'il peut trouver sur le papier.»(Richebois, 2016A). Cet échec est peut-être la 
marque de l'absence d'un besoin essentiel : en matière de littérature, la plus-value symbolique ou pratique de l'œuvre littéraire dans sa version dématérialisée est sans doute trop superficielle pour faire pencher la balance - au contraire du livre scolaire par exemple, plus interactif.

Les observations réalisées ici permettent de constater que dans la commercialisation de ses produits ou dans la représentation symbolique qu'elle fait d'ellemême, l'instance éditoriale traditionnelle exploite le numérique comme une chambre d'écho, un simple moyen de démultiplication de ses pratiques habituelles. En conséquence, elle n'offre pas à l'auteur de véritable moyen d'améliorer sa situation, mais peut au contraire cristalliser les tensions déjà ancrées dans les relations que ces deux entités entretiennent. Rappelons malgré tout qu'Internet est, certes, un espace de partage, mais également un espace partagé et constitue également, de fait, un terrain d'expérimentation.

\subsection{La crise de croyance en l'allégeance de l'auteur}

Comme on l'a dit, l'allégeance de l'auteur (qui est plus encore celle à l'instance éditoriale qu'à l'individu-éditeur singulier) est permise par un verrouillage des statuts et des conditions de développement du livre et ce, en dépit de la grande hétérogénéité des structures. Ces pratiques puisent leur force dans un système de conviction : il s'agit d'instaurer une «croyance » en l'imperméabilité et l'incontestabilité de la nature des rapports entre l'auteur et son éditeur. Toutefois l'espace numérique permet certaines tentatives d'émancipation. L'« autorité » et la « persuasion », comme le rappelle Arendt, sont incompatibles (Arendt, 1972B, p. 146). Nous pouvons nuancer cette réflexion, en affirmant qu'un pouvoir qui n'est pas omnipotent possède de facto des marges, soit des états de non-établissement d'une hiérarchie dans l'intersubjectivité. Persuader les potentiels sujets du pouvoir qu'il est impossible de lui échapper est indispensable à la cohésion du système instauré. L'autorité (comme représentation du pouvoir mais aussi comme exemplification de l'institutionnalisation du pouvoir) consiste également à faire accepter que les rapports n'existent pas au-delà de ces marges, et que ces territoires sont incompatibles avec le principe de reconnaissance. Or, ceci n'est pas un état de fait. Dans ce contexte, les nouveaux outils à disposition sont certes exploités par l'édition traditionnelle, mais créent aussi des zones de liberté, non soumises à leur joug.

Pour l'instance éditoriale, détentrice des clés du pouvoir et en position de force, les nouveaux outils représentent un moyen d'entériner ce pouvoir dont elle disposait déjà, et ceci sans renégocier son ascendant sur l'auteur. Face à cette situation dans laquelle beaucoup ne trouvent pas leur compte, une échappatoire apparaît pourtant, qui 
repose sur l'utilisation des mêmes outils que ceux des instances dominantes. Internet est un lieu partagé par tous ; ainsi, ce dernier offre également des espaces en marge des sphères de contrôle. Ces « zones franches " paraissent, à priori, constituer une alternative auprès des auteurs désireux de trouver ou retrouver une autorité auctoriale qu'ils estiment spoliée, qu'ils n'estiment pas appréciée à sa juste valeur, ou auprès des auteurs en simple quête de visibilité que ne leur donne pas, ou ne peut leur donner le milieu éditorial. Internet offrirait ainsi une chance à tous les exclus et les invisibles, les refusés du système marchand dont l'équilibre repose essentiellement, encore aujourd'hui, sur le sacrifice d'une majorité d'indigents au profit d'une minorité.

\section{Les recherches de nouvelles formes d'autorité auctoriale dans un contexte de mutation}

\subsection{L'auteur auprès des nouveaux réseaux}

Internet comme espace de partage et espace partagé est le moyen pour l'auteur non seulement de produire son œuvre de manière différente, sans passer par les canaux usuels, mais aussi le moyen de construire une image d'auteur différente. L'auteur peut ainsi se bâtir une identité de son propre chef, sans se reposer sur une stratégie spécifiquement éditoriale ; ceci peut prendre différentes formes. L'auteur peut ainsi avoir un compte sur les réseaux sociaux (Facebook et Instagram étant les plus utilisés aujourd'hui) ; blogs et sites d'auteurs représentent également une part non négligeable de l'activité auctoriale en ligne. Ces outils représentent notamment un moyen d'entrer dans un rapport de proximité avec les lecteurs (Wiart, 2019, p. 83) : l'auteur peut, de la sorte, à la fois tisser du lien social indépendamment de toutes les sphères de légitimation, en échangeant directement avec sa communauté. Internet permet ainsi au lecteur l'expression directe de ses attentes; dans un contexte où les instances de prescription sont en pleine mutation, celui-ci devient « actif, réactif, interactif et participatif ", "lecteur-roi » qui «change donc les règles du jeu de la reconnaissance littéraire ». (Ducas, 2014, p. 71). Adrien Mangold, auteur publié aux éditions de l'Homme, déclare ainsi :

Facebook, c'est pour le contact direct avec les lecteurs. [...] Facebook, c'est pour la communication un peu plus quotidienne, même si je n'en fais pas quotidiennement. C'est pour le côté « interactivité avec les gens » propre aux réseaux sociaux, avec tous les avantages et les inconvénients de ces réseaux. (Entretien avec l'auteur)

Mais les réseaux sociaux comme les sites d'auteurs sont aussi un moyen d'assurer sa propre promotion, notamment par l'évocation d'un calendrier de rencontres, 
dédicaces, déplacements, et autres évènements de nature à favoriser l'interaction de l'auteur avec son public, à fortiori si l'éditeur n'est pas actif sur les réseaux, et n'assure pas cette publicité. L'activité auctoriale en ligne varie cependant grandement d'un auteur à l'autre : pour se contenter de deux exemples opposés, si Marc Lévy y est très peu actif, Leila Slimani l'est beaucoup plus. Les réseaux ne sont pas forcément des espaces de création textuelle, mais possiblement juste de mise en scène de soi, d'une posture d'écrivain dématérialisée. Les nouveaux relais sont, enfin, une manière d'affirmer l'autorité d'auteur par l'apport de conseils d'écriture, comme le fait notamment Bernard Werber (Werber, s. d.), ou un espace d'échange avec le lectorat ; le site d'auteur devient aussi possiblement un espace de création et d'expérimentation textuelle, comme le fit Hubert Nyssen, éditeur mais aussi écrivain (Nyssen, s. d. $)^{8}$. Mais bien que ces usages puissent témoigner d'une volonté de regain ou de confirmation de la posture auctoriale, ou d'une mise en place d'une stratégie d'auteur alternative à celle qu'instaure l'instance éditoriale, ils peuvent aussi devenir le reflet de cette instance, voire l'incarner comme blason. En effet, si les comptes sur les réseaux donnent à voir une certaine image d'auteur, comment savoir si ces comptes sont animés par les auteurs eux-mêmes, ou par d'autres acteurs? Internet se conçoit comme un espace où, selon la formule de Klossowski, s'épanouit le simulacre, qui « imite ce qu'il appréhende dans le phantasme » (Klossowski, 1984, p. 77). La présence en ligne peut alors devenir le relais de la mythification à la fois de l'auteur (par l'évocation de la biographie) et de l'éditeur (par la bibliographie), et arborer une dynamique essentiellement promotionnelle, allant jusqu'à rediriger vers des plateformes de vente. Ces modèles, toutefois, sont fréquemment l'apanage des auteurs best-sellers, à l'instar de Guillaume Musso (Musso, s. d.). Derrière le jeu sur l'imaginaire de l'« auteur de proximité ", d'autres sont réduits à des marques. Dans tous les cas, comme le rappelle Sylvie Ducas, « Les réseaux numériques [...] rappellent combien, pour exister, l'écrivain a besoin de ses pairs et des réseaux de sociabilité littéraires. » (Ducas, 2016, p. 646).

La présence numérique de l'auteur décrite ici est essentiellement celle d'écrivains déjà publiés par des structures traditionnelles. Internet n'est exploité que comme espace complémentaire au circuit de l'imprimé. Si ces modèles se construisent en parallèle de l'activité éditoriale en ligne ou en collaboration avec elle, ils n'y sont pas étrangers; d'autres alternatives existent, où l'indépendance s'observe dès l'acte même de création.

8 Encore actif jusqu’à la fin de l'année 2020, le site est aujourd'hui indisponible. 


\subsection{L'autorité autopoïétique par l'exemple des sites d'autoédition}

L'un des exemples les plus significatifs de l'exploitation de ces marges dans la sphère numérique est celui de l'autoédition en ligne, qui se comprend comme l'un de ces espaces tentant, avec plus ou moins de succès, de s'affranchir du joug éditorial ou, à tout le moins, d'offrir une solution de repli à ses usagers n'ayant pu, malgré leurs tentatives, faire allégeance à une maison d'édition. Les plateformes qui permettent ces actions apparaissent avec le changement de millénaire (la première, lulu.com, est inaugurée en 2002), et revêtent aujourd'hui des apparences multiples. Mais qu'elles soient si célèbres qu'elles n'en finissent pas de défrayer la chronique, comme le Kindle Direct Publishing d'Amazon fondé en 2007, ou qu'elles restent beaucoup plus discrètes dans les médias, des caractéristiques communes les définissent. La première et plus essentielle d'entre elles tient en la revendication d'une désintermédiation maximale entre l'auteur et le lecteur : dans une dynamique de rapprochement, toutes les étapes qui les séparent sont remplacées par la plateforme elle-même, qui sert alors de support partagé par les deux maillons encore en place. La directrice de Librinova dit ainsi :

L'idée, c'est que les auteurs puissent trouver chez nous tout ce qu'ils pourraient avoir dans une maison d'édition, et qu'ils puissent le prendre en fonction de ce dont ils ont besoin. En gros, qu'ils puissent faire leur marché : comme dans une maison d'édition, mais qu'ils décident de prendre telle chose et de ne pas prendre telle autre, parce qu'ils peuvent parfois se débrouiller eux-mêmes et parfois ont besoin d'aide. (Entretien avec l'auteur)

En conséquence, une liberté quasi-totale est laissée à l'auteur, qui s'étend à toutes les étapes de la chaîne du livre. Ce dernier, à ce titre, bénéficie de multiples avantages et privilèges revenant usuellement à l'instance éditoriale. Il devient seul décideur de la forme finale du produit, ses choix s'imposant aussi bien sur les aspects diégétiques que paratextuels (couverture, titre, harmonie du texte, etc.). Au-delà des considérations esthétiques, les avantages présentés sont aussi économiques, et relatifs à des questionnements matériels (prix du livre, délais de parution, etc.). L'œuvre est mise « tout de suite sur le marché »(Amazon, s. d.), la publication ne prend que " quelques minutes seulement» (Librinova, s. d. A), loin de l'attente usuelle dans l'imprimé, parfois fonction d'un calendrier sur lequel l'auteur n'a pas de contrôle ${ }^{9}$. La désintermédiation permise et

9 Le modèle-type de contrat d'édition commenté, réalisé par le Conseil Permanent des Écrivains (CPE) et préconisé par la Société des Gens de Lettres (SGDL) recommande ainsi un «délai maximum de huit mois à compter de la remise définitive des éléments permettant la publication». (Société des Gens de Lettres, 2020, p. 16). 
revendiquée par les nouveaux relais devient une manière d'interroger la pertinence des rapports usuels. De fait, elle est fréquemment couplée à un discours de déconsidération de ce système. Si la compétence des acteurs professionnels du milieu n'est pas particulièrement attaquée, ni la légitimité du circuit de l'imprimé en tant que tel (voir infra), c'est le fonctionnement institué lui-même qui est dénoncé, comme à la fois trop sélectif et trop peu rémunérateur. Un argument récurrent, quand la monétisation est possible, porte sur les droits d'auteurs plus élevés - Amazon avancent des taux « qui peuvent atteindre $70 \%$ »(Amazon, s. d.) -, et sur leur opposition aux $10 \%$ en usage dans l'imprimé (Atramenta, s. d.). Libérés des contraintes économiques de logiques dont ils ne détiennent pas les clés mais qu'ils considèrent néanmoins comme contraignantes, affranchis des stratégies auctoriales les laissant de côté (qui sont en réalité aussi bien des stratégies éditoriales), ces auteurs prennent en apparence le contrôle de leur œuvre et de sa commercialisation. L'auteur, dans ce cadre où la plateforme de partage ne joue que le rôle de simple support, ne soumet plus la création à l'approbation d'un tiers. Débarrassée de cette ascendance, l'œuvre autopubliée, donc l'œuvre pour laquelle l'attribut littéraire est désormais attesté par l'auteur et seulement par lui, sans aucun filtre préalable, s'ancre dans une conception autopoiétique de la littérarité. Dans ce contexte, la question même d'autorité n'est plus seulement en crise, mais simplement inexistante, en raison de l'absence des deux prémices nécessaires à sa constitution, à savoir l'intersubjectivité et la hiérarchie. Toute intersubjectivité ne disparaît pas pour autant : l'œuvre reste adressée à un lectorat, qui, tout en gardant son statut de profane, devient, dans sa masse, le moteur de la reconnaissance ultérieure à la publication.

Les formes instituées de la confection de la littérarité et de la légitimité ne sont maintenant plus tenues exclusivement par des institutions qui se reconnaissent ces droits à dire « ce qui est littéraire » et « ce qui est légitime ». Ces autorités sont maintenant réparties en une pluralité d'acteurs, institutionnels ou non, qui revendiquent également une légitimité. L'effet est bien celui d'un d'effondrement : effondrement de la croyance dans le système institué par les auteurs, qui cherchent malgré tout un public et la reconnaissance de leur statut d'écrivain ; « impression » d'effondrement des réseaux traditionnels de construction de la valeur, qui reposaient sur une reconnaissance de la professionnalisation. Ainsi, comme le précise la directrice de Librinova, les réseaux de prescription deviennent désormais aussi des réseaux constitués d'amateurs :

On a vraiment tous les nouveaux médias autour du livre : les bloggeurs, les influenceurs littéraires et tout : ça, ça marche hyper bien. Eux, pour le coup, ils lisent de plus en plus en numérique - parce que les journalistes, eux, ne lisent pas en numérique. (Entretien avec l'auteur) 
Revendiquer sa propre légitimité, et sa légitimité à « dire » ce qui est légitime (soit : définir son propre rôle, et définir celui des autres) se comprend comme une remise en question de l'autorité et de la légitimé des institutions. C'est ainsi qu'un discours récurrent visible sur ces plateformes (ou de la part de leurs porte-paroles) est celui de dire vouloir agir contre, en parallèle, ou à défaut en marge du système éditorial traditionnel (Atramenta, s. d.). Cette liberté apparente n'est toutefois pas exempte de nuances. Considérons tout d'abord que l'autoédition en ligne, comme d'ailleurs le circuit de l'édition imprimée, ne constitue pas un système homogène mais se compose d'une multitude de formes et d'approches dans la constitution et la valorisation des œuvres et de leurs auteurs. Afin de financer leur fonctionnement, certaines de ces plateformes d'autoédition proposent de la sorte tout un panel de services payants (le simple dépôt de l'œuvre sur la plateforme n'étant pas inclus dans ceux-ci), allant de l'accompagnement promotionnel (par la représentation auprès de sites partenaires, etc.) (Librinova, s. d. B), ou de conseils de nature éditoriaux (choix de couverture, relecture, correction, etc.) (IggyBook, s. d.). Plus encore que simples prestataires de services, dès lors que les pratiques de ces nouvelles instances revendiquent une légitimité à " dire le littéraire » (et se supposent, ou se disent légitimes à agir de la sorte) par-delà la parole de l'auteur, ces acteurs présentent des caractéristiques manifestement inspirées des instances au pouvoir. Certains produisent même des discours promouvant les modèles usuels des rapports hiérarchiques. La fondatrice d'IggyBook déclare ainsi : « Notre ADN, c'est d'être éditeurs. » (entretien avec l'auteur). La réplication du schéma traditionnel n'est pas limitée qu'aux logiques de production et de mise à disposition des œuvres, mais possiblement à tout un processus de consécration aussi inspiré des pratiques instituées. Librinova a ainsi fondé en 2016 le Prix des Étoiles, sélectionnant des ouvrages autoédités et récompensant le lauréat d'une garantie de publication imprimée :

Les prix littéraires, c'est super ; mais ce sont les dix mêmes livres qui sont dans toutes les présélections. Le but du Prix des Étoiles, ceétait de récompenser des livres qui n'avaient pas forcément fonctionné dans l'autoédition mais qu'on avait quand même envie de représenter, de pousser, parce qu'il y a quand même des livres super chez nous. (Entretien avec l'auteur)

La recherche de reconnaissance passe, de fait, également par l'exploitation de codes déjà établis. Ces prix, toutefois, peinent encore à acquérir une reconnaissance symbolique, et ne remettent pas en cause le système classique des prix littéraires comme l'illustre l'exemple du Prix Amazon de l'autoédition (Majorel, 2015). Toutefois, certains signaux forts apparaissent et sont les témoins d'une mutation 
dans l'équilibre des instances de consécration littéraire, telle que l'entrée dans la sélection Renaudot de 2018 de Marco Koskas, auteur autoédité, à l'origine d'une vaste polémique (Oury, 2018). Par ces divers procédés, ces plateformes cherchent à acquérir un certain capital symbolique, qui n'est autre que celui qui se construit dans le circuit conventionnel. Autrement dit, les plateformes dont il est question copient les principes hiérarchiques et réticulaires des structures éditoriales traditionnelles pour mieux les concurrencer ou s'adressent directement à elles et, à ce titre, ne font que renforcer le bien-fondé des processus propres à ces structures. Ces plateformes ne se positionnent donc pas dans une logique de révolution des modèles (soit de transformation radicale du système de valorisation de la littérature et de ce qui la produit et la promeut), mais plus volontiers dans une logique de reproduction de ces modèles. Il ne s'agit donc, pour l'heure tout du moins, que d'une simple alternative, une forme encore atténuée de concurrence qui n'entraîne qu'un affaiblissement relatif du système traditionnel.

Cette inspiration partielle des modèles traditionnels (soit : permettre de faire de livre, et de faire vivre le livre) est aussi un moyen d'attirer les auteurs. Dans les faits, l'adresse d'un auteur à ces nouveaux relais est majoritairement résumable en deux grands ensembles. Elle peut être d'abord motivée par un désespoir, suite aux multiples refus éditoriaux des manuscrits proposés, et faire alors figure de dernier recours, ce que rappellent fréquemment les argumentaires de ces plateformes (Desseilligny, 2017, p. 120). Le directeur du site MonBestSeller affirme ainsi :

On est partis d'un constat, c'est qu'en fait, il y avait plein d'écrits qui, d'abord, passaient inaperçus, même de ceux qui étaient édités, et surtout, il y avait $99 \%$ des gens qui nétaient pas édités. On s'est dit que dans ces $99 \%$, c'était impossible qu'il n'y ait pas, un) de bons ouvrages, et deux) - c'est surtout de là qu'est partie notre réflexion -, pourquoi est-ce que l'édition, le monde littéraire, etc. restaient dans cette tour d'ivoire, à mépriser tous ceux qui écrivent. (Entretien avec l'auteur)

Elle peut aussi être motivée par un désir d'expérimentation dans l'exploitation de formes textuelles déjà délégitimées par l'édition traditionnelle. C'est un des facteurs expliquant la grande popularité de la littérature de genre sur ces plateformes (Noëson, 2016). Sur nombre d'aspects, les pratiques numériques dites "alternatives " ne sont donc qu'une chambre d'écho de ce qui se constate d'ores et déjà ailleurs, mais c'est aussi cette proximité qui permet aux auteurs en puissance de se tourner vers elles. Il s'agit maintenant de chercher à observer si ces phénomènes ont un impact notable sur le pouvoir institué et ses pratiques. 


\subsection{Les limites à ces pratiques : la puissance du système traditionnel}

Les marques concrètes de la possession du pouvoir par les institutions traditionnelles sont, d'une part, la capacité essentielle d'agir comme premier filtre de la production et, d'autre part, le contrôle effectif du marché du livre. Du point de vue économique, l'impact des pratiques alternatives n'est que modéré ; en conséquence, l'autorité de ceux qui agissent au nom du pouvoir n'est pas réellement fragilisée. Le PDG de Hachette a à cœur de le rappeler : «L'autoédition ne constitue pas une menace.» (Vulser, 2018). Comme les chiffres mentionnés supra le démontraient, l'impact du numérique sur l'économie du livre reste encore globalement négligeable. Les nouvelles pratiques, qui s'inscrivent dans cette économie alternative, ne forment pas une menace pour la puissance mercantile du système français, ni ne fragilisent le marché de l'imprimé. Cette absence de menace n'empêche pas malgré tout les instances traditionnelles d'être globalement critiques envers ces nouveaux moyens de création et leurs utilisateurs. Pour ne prendre qu'un exemple, la Société française des Intérêts des auteurs de l'écrit (Sofia), indique clairement sur son site officiel : «Les auteurs autoédités ne peuvent pas adhérer à Sofia car ils ne remplissent pas la condition d'un réel contrat d'édition, nul ne pouvant, en droit français, contracter envers soi-même [...] (Société française des Intérêts des auteurs de l'écrit, s. d.).

Cette déconsidération de la part des institutions devient alors un moyen de valoriser le schéma traditionnel. En effet, si le but originel de la Sofia est de défendre les droits d'auteurs et, dans une certaine mesure, de tempérer la précarisation que ces auteurs peuvent rencontrer, il apparaît que ces aides ne peuvent être versées qu'à la condition que ceux-ci soient subordonnés à l'institution éditoriale. Les acteurs euxmêmes de cette institution, plutôt que de voir dans ces nouvelles pratiques une remise en cause du bien-fondé de leur statut, en profitent volontiers pour affirmer leur propre légitimité. La déconsidération des nouvelles formes éditoriales repose ainsi également sur l'affirmation, en opposition, d'un savoir-faire qui découle naturellement de la professionnalisation des statuts, mais aussi de la connaissance du marché. Un éditeur des Presses de la Cité dit ainsi :

L'argument du droit de chacun à être vu, être publié, c'est la prime à l'amateurisme, et si je chante sous ma douche, dois-je m'insurger de ne pas passer à l'Olympia ? C'est faire bien peu de cas, me semble-t-il, du travail de l'éditeur, de sa fonction même. (Entretien avec l'auteur)

La valorisation de l’ouvre littéraire devrait donc passer par un éditeur traditionnel pour que celle-ci soit « accomplie »; cet éditeur deviendrait dès lors une garan- 
tie de validité. Bien entendu, ce discours n'est pas représentatif de la totalité des acteurs du livre ; si cette déconsidération est un phénomène fréquent, elle n’empêche pas malgré tout les acteurs traditionnels d'établir un ensemble de liens avec ces nouveaux modèles. Ainsi, ces considérations doivent être nuancées au regard des phénomènes de récupération d'auteurs à succès en provenance du domaine de l'autoédition. Les cas sont rares mais restent emblématiques; en France, deux noms reviennent fréquemment dans la presse : Aurélie Valognes, et Agnès Martin-Lugand, publiées aujourd'hui respectivement chez Fayard et Michel Lafon, ce dernier étant par ailleurs familier de ces pratiques (Bessard-Banquy, 2017). Dans ce processus, l'œuvre est extraite du circuit de l'autoédition pour rejoindre les bancs du circuit classique, phénomène participant à renforcer la portée symbolique attachée à l'édition traditionnelle. Celle-ci paraît alors plus que jamais perçue comme un vecteur de légitimation plus fort et mieux ancré que l'autoédition, ce qui n'amène qu'à percevoir encore plus cette dernière comme une zone de transition avant l'accès à la « véritable » reconnaissance littéraire, et au statut de «véritable» écrivain aux yeux des instances de légitimation. À l'opposé du discours promouvant l'indépendance vis-à-vis du système, plusieurs de ces plateformes avancent même une valorisation revendiquée du circuit de l'imprimé. Dans l'objectif assumé de favoriser ces récupérations, des affinités peuvent être tissées entre entreprises d'autoédition et structures traditionnelles, les premières se définissant dès lors comme des antichambres de la légitimité. La fondatrice de Librinova déclare ainsi : «C'était ça, notre souhait de départ [...] : de se servir de tout cela comme d'un tremplin pour en amener certains - le plus possible - vers l'édition traditionnelle. On pourrait dire que l'autoédition serait un outil-test pour les éditeurs. » (entretien avec l'auteur).

L'argument n'est ici plus de se substituer ou de remplacer des modèles anciens par d'autres, plus récents, en marge des premiers ; il ne s'agit, au mieux, que d'acquérir assez de légitimité pour les égaler ou les concurrencer. L'accès à la reconnaissance de ces plateformes ne va pourtant pas de soi. Ces outils restent bel et bien " en marge » et si des ponts existent bel et bien, induisant une prise en compte croissante des nouveaux apports par les sphères traditionnelles, ces ponts ne bénéficient une fois de plus qu'à de rares élus. L'imposante majorité des créateurs demeurent dans l'ombre, dans un entassement de productions dénué de tout filtre ; à cet égard, peut-être l'invisibilité est-elle encore plus flagrante que dans le circuit classique. La récupération d'œuvres en provenance de l'autoédition, malgré tout, peut contribuer à fragiliser l'image de l'éditeur traditionnel : en échange d'un risque éditorial moindre, l'éditeur ne fait plus office de découvreur de talents mais se réduit à un simple label qualité cautionnant le succès numérique (Richebois, 2016B). 


\section{Conclusion. Une crise somme toute assez relative à l'heure actuelle, et des tentatives dont la portée n'est pas assez prégnante pour déstabili- ser le système institué actuel}

La « crise » dont il est question est donc le syndrome de l'effondrement d'une confiance de l'auteur envers l'éditeur, mais aussi de l'effondrement en la croyance du bien-fondé d'un système établi. La soumission de l'auteur, effective depuis déjà plus de deux siècles, se poursuit manifestement encore aujourd'hui. Le désir d'émancipation, s'il est possible, laisse fondamentalement apparaître un double écueil : le premier est relatif à la reconnaissance de l’œuvre comme « œuvre littéraire »; le second est relatif à l'acquisition du statut symbolique d'« écrivain ». Bien que les pratiques traditionnelles soient elles-mêmes en mutation, leur emprise symbolique reste donc suffisamment forte pour que la reconnaissance des nouvelles pratiques par ce même système établi ne soit qu'encore (très) modérée. Le verrouillage du marché du livre est si inébranlable que la recherche de visibilité dans l'espace numérique ne se construit sans doute qu'aux franges d'un ensemble d'ores et déjà dominé par les acteurs institués, en raison de leur capital symbolique dépassant les frontières de la matérialité, mais aussi de leur exploitation de l'espace dématérialisé. Les alternatives que permettent les divers usages du numérique restent en conséquence des pratiques marginales, et font que le capital symbolique de l'édition traditionnelle reste encore trop présent pour être véritablement fragilisé. Peut-être faut-il voir malgré tout, dans ces phénomènes, les prémices de mutations plus fortes à venir, plus dangereuses pour ce système : la crise sanitaire contemporaine a participé à grandement favoriser le recours aux supports dématérialisés. Les comportements récents témoignent d'un intérêt sans cesse croissant pour la lecture en ligne et l'autoédition, contexte particulièrement favorable au développement des structures qui la promeuvent (Guyenne, 2021).

\section{BIBLIOGRAPHIE}

Amazon (s. d.) Publiez, sans frais, des ebooks et livres brochés en autoédition via Kindle Direct

Publishing et touchez des millions de lecteurs sur Amazon. https://kdp.amazon.com/fr_FR/

Arendt, H. (1972A). La Crise de la culture. Gallimard.

Arendt, H. (1972B). Du mensonge à la violence. Essais de politique contemporaine. Calmann-Lévy. Arendt, H. (1998). The Human Condition. The University of Chicago Press.

Atramenta (s. d.). A propos d'Atramenta. http://atramenta.net/about.php

Benhamou, F. (2003). Concurrence pour la table du libraire. Esprit, 6, 98-115.

Bessard-Banquy, O. (2017). De la toile en ligne et inversement. Fabula/Les colloques, Internet est un cheval de Troie. http://fabula.org/colloques/document4187.php 
Bourdieu, P. (1991). Le champ littéraire. Actes de la recherche en sciences sociales, 89, 3-46. https:// doi.org/10.3406/arss.1991.2986

Bouvaist, J.-M. (1991). Pratiques et métiers de l'édition. Editions du Cercle de la Librairie.

Deguy, B. (1988). Le Comité: confessions d'un lecteur de grande maison. Champs Vallon.

Desseilligny, O. (2017). Reformuler les processus éditoriaux, déplacer l'imaginaire du bestseller? Formes, conditions et mythologies du succès en contexte numérique. Revue critique de fixxion française contemporaine, 15, 118-129. http://www.revue-critique-defixxion-francaise-contemporaine.org/rcffc/article/view/fx15.10

Dosse, F. (2014). Les Hommes de l'ombre:portraits d'éditeurs. Perrin.

Ducas, S. (2014). Ce que font les prix à la littérature. Communication et langages, 179(1), 61-73. https://doi.org/10.3917/comla.179.0061

Ducas, S. (2016). Lécrivain contemporain en réseau web 2.0: retour du refoulé auctorial? Revue d'histoire littéraire de la France, 116(3), 641-651. http://www.jstor.org/ stable/24890584

Éditions Actes Sud (s. d.). Page de présentation. http://actes-sud.fr/presentation

Éditions du Seuil (s. d.). Frères sorcières, Antoine Volodine. http://seuil.com/ouvrage/freressorcieres-antoine-volodine/9782021363753

Éditions Fayard (s. d.). Notre histoire. http://fayard.fr/notre-histoire

Éditions Gallimard (s. d.). Marcel Proust. Euvres complètes (tome I, volume I):A la recherche du temps perdu. http://gallimard.fr/Catalogue/GALLIMARD/In-octavo-a-la-gerbe/ OEuvres-completes19

Genette, G. (1971). Seuils. Editions du Seuil.

Guyenne, L. (2021). Au royaume du Covid, l'écriture est reine. France culture. https://www. franceculture.fr/litterature/au-royaume-du-covid-lecriture-est-reine

IggyBook (s. d.). Design de couverture. https://www.iggybook.com/iggystudio/design-decouverture

Jakobson, R. (1973). Questions de poétique. Editions du Seuil.

Jaucourt, L. Pouvoir (1765). Dans Diderot, D. et D'Alembert, J. (dirs.) L'Encyclopédie ou Dictionnaire raisonné des sciences et des métiers, par une société de gens de lettres (vol. XIII, p. 255). Samuel Faulche \& Compagnie.

Jauss, H. R. (1978). Pour une esthétique de la réception. Gallimard.

Klossowski, P. (1984). La Ressemblance. Ryoân-ji.

Librinova (s. d. A). Bienvenue dans notre outil de publication! http://librinova.com/je-me-publie

Librinova (s. d. B). Services promotionnels. https://www.librinova.com/offre/services-a-lacarte/services-promotionnels

Majorel, A. (2015, 29 octobre). Quelle est la valeur du prix littéraire d'Amazon? L'Usine digitale. https://www.usine-digitale.fr/article/quelle-est-la-valeur-du-prix-litteraire-d-amazon. N359375

Musso, G. (s. d.). Mes romans. http://guillaumemusso.com/les-romans-0

Nemer, M. (1996). Les rapports auteur/éditeur. Dans G. Chamarat \& A. Goulet (dirs.). L'Auteur (pp. 185-190). Presses Universitaires de Caen. https://doi.org/10.4000/books. puc.9904 
Noëson, G. (2016, 23 mai). La littérature de genre va-t-elle redistribuer les cartes de l'édition et de la librairie? Lettres numériques. http://lettresnumeriques.be/2016/05/23/lalitterature-de-genre-va-t-elle-redistribuer-les-cartes-de-ledition-et-de-la-librairie/

Nyssen, H. (s. d.) Carnets. http://wwww.hubertnyssen.com/carnets.php

Oury, A. (2014, 3 septembre). Vente directe et soutien aux librairies, la stratégie HarperCollins. Actualitté. http://actualitte.com/article/47161/distribution/vente-directe-et-soutienaux-librairies-la-strategie-harpercollins

Oury, A. (2018, 14 septembre). Renaudot:Marco Koskas dénonce «l'hypocrisie de l'argument» des libraires. Actualitté. https://actualitte.com/article/17175/distribution/renaud otmarco-koskas-denonce-l-hypocrisie-de-l-argument-des-libraires

Peltier, S., \& Moreau, F. (2016). Le Net va-t-il avoir raison des intermédiaires? Nectart, 1(1), 130136. https://doi.org/10.3917/nect.002.0130

Piault, F. (1992). De la «rationalisation»à l'hyperconcentration. Dans Fouché, P. (dir.) L'Edition française depuis 1945 (pp. 629-639). Editions du Cercle de la Librairie.

Piault, F. (2018). Les 200 premiers éditeurs français : classement 2018. Livres Hebdo, (1179), 20-29.

Politis Boublis, K. (2019). L'édition numérique. Dans Les chiffres de l'édition 2018-2019:rapport statistique du SNE. Syndicat National de l'Edition.

Quelquejeu, B. (2001). La nature du pouvoir selon Hannah Arendt: Du 'pouvoir-sur' au 'pouvoiren-commun'. Revue des sciences philosophiques et théologiques, 3(3), 511-527. https://doi. org/10.3917/rspt.853.0511

Reynaud-Cressent, B. (1982). La dynamique d'un oligopole avec frange :le cas de la branche d'édition de livres en France. Revue d'économie industrielle, 22, 61-71. https://doi. org/10.3406/rei.1982.2072

Richebois, V. (2016A, 19 juin). Hachette investit les jeux vidéos avec Neon Play. Les Echos. http://www.lesechos.fr/amp/209481

Richebois, V. (2016B, 18 mai). L'autoédition, nouveau terrain de chasse des éditeurs. Les Echos. https://www.lesechos.fr/2016/05/lautoedition-nouveau-terrain-de-chasse-desediteurs-229669

Sadowska-Guillon, I. (1988). La voie de la découverte de Hubert Nyssen:Les éditions Actes Sud. Jeu:revue de thêâtre, (48), 120-128. https://id.erudit.org/iderudit/28354ac

Société des Gens de Lettres (2020). Contrat d'édition commenté. https://sgdl.org/ phocadownload/Juridique/2020/Contrat_comment\%C3\%A9_Juillet_2020.pdf

Société française des Intérêts des auteurs de l'écrit (s. d.) Foire aux questions. http://www.lasofia.org/vos-questions/

Sintomer, Y. (1994). Pouvoir et autorité chez Hannah Arendt. L'Homme et la société, (113), 117131. https://doi.org/10.3406/homso.1994.2756

Syndicat National de l'Edition (2000). Convention collective nationale de l'Edition. https://www. sne.fr/app/uploads/2017/11/ConventionCollective.pdf

Viala, A. (1985). Naissance de l'écrivain. Minuit.

Vulser, N. (2018, 10 octobre). Arnaud Nourry, PDG d'Hachette Livre:«L'autoédition ne constitue pas une menace».Le Monde. https://www.lemonde.fr/economie/ article/2018/10/10//-autoedition-ne-constitue-pas-une-menace_5367245_3234.html

Weber, M. (1995). Economie et Société, tome 1:Les catégories de la sociologie. Pocket. 
Werber, B. (s. d.). Conseils aux écrivains. http://www.bernardwerber.com/unpeuplus/conseils_ ecrivains.php

Wiart, L. (2019). Le personal branding des écrivains sur les réseaux sociaux: gestion de l'identité et de la notoriété en ligne. Communiquer, 26, 67-87. https://doi.org/10.4000/ communiquer.4220

Après un Master en littérature comparée obtenu à l'université Rennes 2, et suite à une formation au Pôle Métiers du Livre de Saint-Cloud, Corentin Boutoux a travaillé dans plusieurs secteurs du milieu éditorial (universitaire, scolaire, généraliste, et scientifique) à partir de 2014. Il poursuit actuellement une thèse au Centre des Sciences des Littératures en langue Française (CSLF, EA 1586) à l'Université Paris Nanterre, sous la direction de Sylvie Ducas. Ses recherches portent sur « Les nouvelles formes d'autorité dans les siècles du contemporain $\left(\mathrm{XX}^{\mathrm{e}}-\mathrm{XXI} e\right)$ : l'exemple de la relation entre auteurs et éditeurs littéraires. » 